\title{
Uso do óxido nítrico inalatório no tratamento da crise hipertensiva pulmonar no pós-operatório de transplante cardíaco
}

Juan Alberto Cosquillo MEJIA*,**, João David de SOUZA NETO*, Waldemiro CARVALHO JR*,**, Valdester Cavalcante PINTO JR. ***, Fernando Antônio MESQUITA****, Haroldo Barroso BRASIL*,**, Rogean Rodrigues NUNES**, Ítalo Martins de OLIVEIRA***, João Martins de Souza TORRES*

Trabalho realizado na Unidade de Transplante e Insuficiência Cardíaca do Hospital de Messejana. Fortaleza, CE, Brasil. Recebido para publicação em março de 2002. - *Do Hospital do Coração de Messejana. - **Da Clínica Cirucárdio. - *** Da Universidade Federal do Ceará/ bolsista do PIBIC-CNPq.

RBCCV 44205-594

\section{Resumo}

Objetivo: Este trabalho relata a experiência no manuseio da crise hipertensiva pulmonar (CHP) refratária, com o uso do óxido nítrico inalatório (NOi) no pós-operatório imediato do transplante cardíaco (TC) ortotópico.

Métodos: De outubro/1997 a fevereiro/2002 foram realizados $31 \mathrm{TC}$ em adultos, sendo incluídos pacientes com RVP $<6 \mathrm{uW}$, ou $<2,5 \mathrm{uW}$ na prova farmacológica. Após o período de circulação extracorpórea (CEC) $(\mathrm{M}=101 \pm 21$ minutos), todos fizeram uso de dobutamina, dopamina e milrinona; entretanto, frente à ausência de resposta adequada e CHP, administrou-se NOi em doses crescentes de 20 a 40ppm. Foram utilizados registros das pressões através de cateter no átrio esquerdo e no tronco da artéria pulmonar, gasometria arterial seriada e ecocardiograma transtorácico (ETT). $O$ tempo médio de morte encefálica (ME) do doador foi de 16 $\pm 5,1$ horas.

Resultados: Cinco pacientes (1 mulher), com idade média de 42 anos, fizeram uso de NOi por apresentarem critérios de CHP, todos com sinais de baixo débito cardíaco. $O$ tempo médio de CEC foi de $150,8 \pm 34,3$ minutos, a média sistólica arterial pulmonar foi de $87 \mathrm{mmHg}(75-115)$ e a $\mathrm{PO} 2$ média de $60 \mathrm{mmHg}(\mathrm{FiO} 2100 \%)$, sendo evidenciada dilatação moderada a severa do ventrículo direito no ETT. Foi administrado NOi durante uma média 35h (6-96), iniciando-se desmame após estabilização hemodinâmica. $O$ tempo médio de ME do doador foi de 27,6 \pm 5 ,5horas, a internação hospitalar média foi de 63 dias (17-145), uma morte ocorreu por sepse no $17^{\circ}$ PO e os demais pacientes estão em CF I (NYHA).

Conclusões: $O$ tempo de CEC superior a 120 minutos e tempo de ME do doador superior a 20 horas sugerem fatores de risco para CHP no período pós-operatório imediato do TC.
O NOi é uma ferramenta útil no manuseio desta complicação, refratária ao tratamento convencional no POI de pacientes submetidos ao TC.

Descritores: Óxido nítrico, uso terapêutico. Transplante cardíaco. Disfunção ventricular direita. Hipertensão pulmonar.

\section{Abstract}

Objective: This study reviews our experience with the use of nitric oxide inhalation (NOi) in the treatment of the refractory pulmonary hypertensive crisis (PHC) during the immediate post-operative period (IPOP) of heart transplantation (HT).

Method: Between Oct/97 and Feb/02, 31 HT in adults were performed. Among them were patients with Pulmonary Vascular Resistance (PVR) greater than $6 \mathrm{uW}$, or patients with PVR greater than $2,5 \mathrm{uW}$ during the pharmacological test. All patients were weaned from the CPB $(M=101 \pm 21$ minutes) in use of dobutamine, dopamine and milrinone. In the absence of an adequate response to these drugs and with the evidence of PHC, NOi was given, beginning with 20ppm and increasing, when necessary, up to $40 \mathrm{ppm}$. The recording of the left atrium and pulmonary artery pressures, serial arterial blood gases samples and trans-thoracic echocardiograms (TTE) were performed. The donor's brain death (BD) time mean was $16 \pm 5,1$ hours.

Results: In five patients ( 1 female) (mean age $=42$ years) NOi was employed, due to low cardiac output signs and evidence of PHC and right ventricular dysfunction. The

Endereço para correspondência: Juan Alberto Cosquillo Mejia. 
mean CPB time was $150,8 \pm 34,3$ min. The pre-NOi mean PAP was $87 \mathrm{mmHg}$ (range 75-115), and the mean arterial PO2 = $60 \mathrm{mmHg}(\mathrm{FiO2}=100 \%)$. The TTE showed moderate-severe dilation of the right ventricle. NOi was given during a mean of $35 \mathrm{~h}$ (range 6 to 96), being weaned only after hemodynamic stability was achieved. There was only one early death, at the 17 th post op. day due to sepsis. All the remaining patients are in NYHA class $I$.

\section{INTRODUÇÃO}

A falência primária do enxerto após o transplante cardíaco ortotópico é um evento de difícil manejo clínico e confere um importante índice de mortalidade precoce. Fatores como o tempo prolongado de isquemia do enxerto, a injúria de reperfusão e, principalmente, história de hipertensão pulmonar fixa, provocam a falência ventricular direita, com consequiente hipotensão e choque, sendo responsável por $50 \%$ das complicações graves e $19 \%$ dos óbitos no pósoperatório do transplante cardíaco ortotópico ${ }^{(1,2)}$.

Drogas vasoativas como o isoproterenol e o nitroprussiato de sódio (NTPNa) são úteis no tratamento da hipertensão arterial pulmonar, entretanto, frente aos limitantes efeitos sistêmicos e à refratariedade em alguns casos, o óxido nítrico inalatório (NOi) vem se tornando uma ferramenta útil em episódios mais graves, reduzindo a pressão arterial pulmonar e melhorando, consequentemente, a performance ventricular direita ${ }^{(3)}$.

A ação do óxido nítrico (NO) se justifica por ser um gás que, na forma inalatória, se comporta como um vasodilatador pulmonar seletivo. O NO dentro do alvéolo se difunde rapidamente através da membrana alvéolo-capilar, provocando o relaxamento da musculatura lisa do vaso pulmonar.

Deste modo, o presente estudo descreve e avalia o uso do NOi em 5 pacientes submetidos ao transplante cardíaco ortotópico que desenvolveram crise hipertensiva pulmonar (CHP) refratária aos vasodilatadores endovenosos convencionais.

\section{MÉTODO}

\section{População}

De outubro de 1997 a fevereiro de 2002, foram realizados 31 transplantes cardíacos em adultos na Unidade de Transplante e Insuficiência Cardíaca do Hospital de Messejana, Fortaleza, Ceará.

Todos os pacientes foram submetidos a uma avaliação hemodinâmica prévia das pressões e resistências pulmonares e sistêmicas, sendo incluídos na lista de espera do Transplante Cardíaco pacientes com resistência vascular
Conclusions: The prolonged CPB time (> $120 \mathrm{~min}$ ) and the time of brain death of the donor are factors that increase the risk of PHC. The NOi is a useful tool in dealing with this complication when there is an inadequate response to the conventional treatment.

Descriptors: Nitric oxide, therapeutic use. Heart transplantation. Right ventricular dysfunction. Pulmonary hypertension.

pulmonar menor que 6 unidades Wood $(\mathrm{uW})$ no primeiro registro, ou 2,5uW após prova farmacológica com o uso do nitroprussiato de sódio. Na persistência da hiper-resistência pulmonar, foi administrado NOi em baixas doses (20-40ppm), sendo novamente excluídos pacientes que permaneciam com hiper-resistência pulmonar ${ }^{(7)}$.

O tempo médio entre o completo diagnóstico de morte encefálica do doador (segunda avaliação) e o transplante foi de $16 \pm 5,1$ horas e o tempo médio da circulação extracorpórea foi de $101 \pm 21$ minutos.

A técnica cirúrgica utilizada foi a ortotópica bicaval unipulmonar em todos os pacientes. Após a conclusão da circulação extracorpórea, de acordo com a indicação, fizeram uso de isoproterenol (até $0,5 \mu \mathrm{g} / \mathrm{kg} / \mathrm{min}$ ), dobutamina, dopamina ou milrinona.

Na suspeita de CHP e disfunção ventricular direita (DVD), foram colocados cateteres para registro de pressões na artéria pulmonar e átrio esquerdo e melhor avaliação hemodinâmica. Nos casos de vigência de crise hipertensiva pulmonar (pressão arterial pulmonar sistólica 'PAPs' > $60 \mathrm{mmHg}$ ), foi realizada avaliação da anastomose da artéria pulmonar, procurando alterações que provocassem gradiente importante com o ventrículo direito. Também foi descartada a possibilidade de embolia gasosa coronariana e pulmonar ou reação à protamina, bem como adicionadas ou acrescentadas doses de vasodilatadores pulmonares e inotrópicos, sendo, nos casos refratários, iniciada a administração do NOi.

\section{Critérios de Inclusão e Utilização do Noi}

Foi administrado NOi em cinco pacientes frente à ausência de resposta adequada à terapia empregada e persistência de crise hipertensiva pulmonar severa (PAPs > 60mmHg) e DVD secundária. Todos tinham sinais de baixo débito cardíaco, oligúria e hipotensão. Ao ecocardiograma, seguindo às diretrizes da Tabela 1 , foram evidenciados átrio esquerdo normal ou diminuído, ausência de gradiente transpulmonar e dilatação do ventrículo direito com visualização de sua via de saída.

Um cilindro contendo 500ppm de NO em nitrogênio (N2) (White-Martins, Ceará, Brasil) foi conectado a um misturador de gases. A mistura de NO e N2 foi continuamente realizada 
através do ar inalado e a monitorização da mistura e do ar exalado.

foi realizada por dois analisadores eletroquímicos posicionados próximo à cânula traqueal, visando à dosagem das concentrações de $\mathrm{NO}$ e dióxido de nitrogênio (NO2) Figura 2.

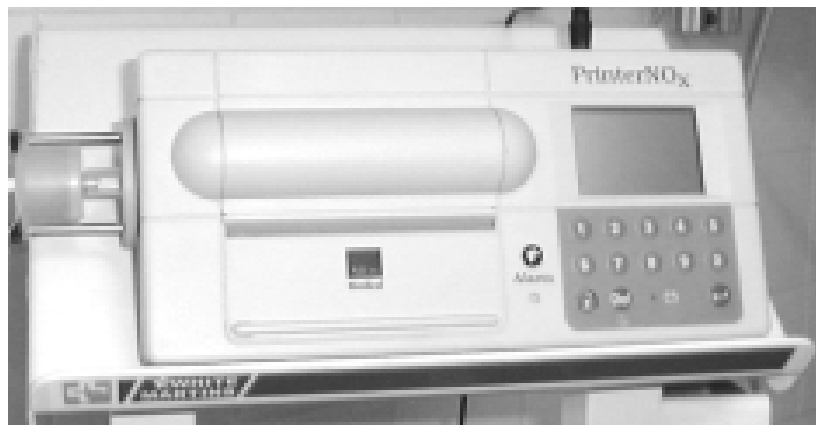

Fig. 2 - Monitorização do $\mathrm{NO}$ administrado e do $\mathrm{NO}_{2}$ exalado (PrinterNOx - White Martins).

A administração de NO foi iniciada com uma dose fixa de 20 partes por milhão (ppm) e, frente à persistência de valores elevados de pressão arterial pulmonar, foram administradas doses crescentes até o limite de 40ppm.

Após a estabilização hemodinâmica, com adequação dos valores hemodinâmicos pulmonares e sistêmicos, foi feito desmame do NOi, com retirada gradual em 2ppm/hora.

\section{Monitorização}

Além da medida das pressões do átrio esquerdo e artéria pulmonar, todos os pacientes foram monitorados com ecocardiograma transtorácico seriado de rotina no pósoperatório. - Figura 3.

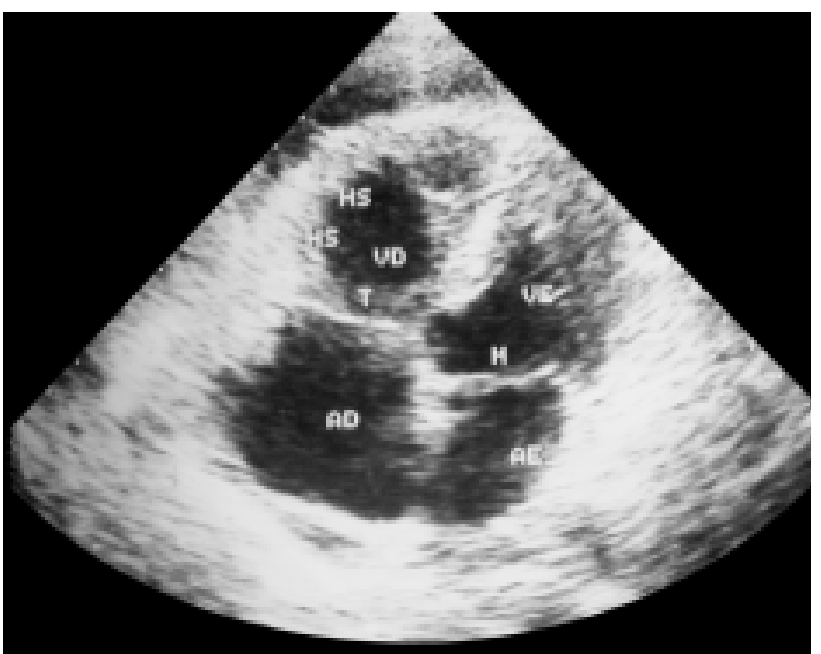

Fig. 3 - Imagem ecocardiográfica demonstrando dilatação severa do ventrículo direito secundária à hipertensão pulmonar.

A toxicidade do óxido nítrico foi controlada através da fração expirada de NO e gasometria arterial seriada com dosagem da metahemoglobinemia, visando manter valores abaixo de $3 \%$.

\section{Análise Estatística}

Os dados são expressos em média \pm desvio-padrão. Para a análise de significância, foi realizado o teste t de Student para dados contínuos não-emparelhados e Wilcoxon Rank Sun. Um valor de p menor que 0,05 foi considerado como estatisticamente significante.

\section{RESULTADOS}

Cinco pacientes, sendo uma mulher, com idade entre 34 e 53 anos (44 \pm 10$)$ fizeram uso de NOi por apresentarem critérios de hipertensão pulmonar severa e DVD, todos com sinais de baixo débito cardíaco.

\section{Dados dos Pacientes}

A FE pré-operatória dos pacientes variou entre $0,13 \mathrm{e}$ $0,29(0,22 \pm 0,06)$, sendo o diâmetro do átrio esquerdo entre $39 \mathrm{e} 48 \mathrm{~mm}$.

$\mathrm{Na}$ avaliação hemodinâmica, as medidas da função cardiovascular se deram de acordo com a Tabela 1.

Tabela 1. Dados pré-operatórios dos pacientes

\begin{tabular}{cccccc}
\hline PACIENTE & FE & IM & IT & IC & RVP \\
\hline 1 & 0,13 & + & + & 2,3 & 4,2 \\
2 & 0,29 & + & + & 2,1 & 2,8 \\
3 & 0,23 & - & - & 2,2 & 2,2 \\
4 & 0,24 & + & + & 1,8 & 4,8 \\
5 & 0,21 & - & - & 1,6 & 6,6 \\
\hline
\end{tabular}

Fração de ejeção (FE); insuficiência mitral (IM) + a 4+; insuficiência tricúspide (IT) + a 4+; índice cardíaco (IC); resistência vascular pulmonar sem drogas uW (RVP); pressão arterial pulmonar sistólica $\mathrm{mmHg}$ (PAPs)

O paciente 5, por apresentar hiper-resistência vascular pulmonar, foi submetido à prova farmacológica com NTPNa, obtendo valores abaixo de 2,5uW, sendo incluído na lista de transplante cardíaco.

Dentre os 5 pacientes estudados, o tempo médio de circulação extracorpórea (CEC) foi de 150,8 $\pm 34,3$ min e o tempo médio de anóxia foi de 103,4 $\pm 19,7 \mathrm{~min}$. O tempo médio entre a morte encefálica (segunda avaliação) e o início do transplante foi de 27,6 $\pm 5,5$ horas - Tabela 2 .

Tabela 2. Dados cirúrgicos dos pacientes

\begin{tabular}{cccc}
\hline PACIENTE & $\begin{array}{c}\text { TEMPO DE CEC } \\
\text { (MIN) }\end{array}$ & $\begin{array}{c}\text { TEMPO DE ANÓXIA } \\
\text { (MIN) }\end{array}$ & $\begin{array}{c}\text { TME } \\
(\mathbf{H})\end{array}$ \\
\hline 1 & 172 & 120 & 21 \\
2 & 131 & 93 & 24 \\
3 & 96 & 81 & 36 \\
4 & 165 & 133 & 25 \\
5 & 160 & 90 & 32 \\
DEMAIS (28) & $101 \pm 21^{*}$ & $90 \pm 16^{\text {**}}$ & $16 \pm 5,1^{\text {** }}$
\end{tabular}

TME - tempo entre o diagnóstico de morte encefálica e o transplante em horas (T ME). * $\mathrm{p}<0,001 ; * * \mathrm{p}=0,105 ; * * * \mathrm{p}<0,001$. 


\section{Dados Pós-Operatórios}

Os dados de pressão da artéria pulmonar, pressão do átrio esquerdo, dosagem de drogas e dilatação do ventrículo direito durante o pós-operatório imediato dos pacientes estão presentes na Tabela 3 .

Tabela 3. Dados pós-operatórios dos pacientes

\begin{tabular}{cccccc}
\hline PACIENTE & PAPs & $\uparrow$ VD & PAE & DOBUTA & DOPA \\
\hline 1 & 115 & +++ & 8 & 15 & 10 \\
2 & 95 & ++++ & 7 & 7,5 & 2,3 \\
3 & 60 & +++ & 7 & 10 & 5 \\
4 & 90 & ++++ & 15 & 14 & 12 \\
5 & 60 & ++++ & 9 & 15 & 15 \\
\hline
\end{tabular}

Pressão Sistólica da artéria pulmonar mmHg (PAPs), dilatação de ventrículo direito ao ecocardiograma + a 4+ (-VD), pressão arterial média mmHg (PAM), dobutamina $\mu \mathrm{g} / \mathrm{Kg} / \mathrm{min}$ (dobuta), dopamina ìg/kg/min (dopa).

A média da pressão sistólica arterial pulmonar foi de $84 \pm$ 21mmHg (60-115) e a PO2 média de 60mmHg (FiO2 100\%), sendo evidenciada dilatação moderada a severa do ventrículo direito ao ecocardiograma transtorácico. Com isso, foi administrado NOi durante 6 a 96 horas $(35 \pm 35)$, iniciando-se desmame de aproximadamente $2 \mathrm{ppm}$ por hora após estabilização hemodinâmica.

Em um caso, a interrupção abrupta acidental de 10ppm no final do curso de um desmame provocou instabilidade hemodinâmica, sendo necessária a readministração de doses mais altas (15 ppm). Após a recuperação a níveis atingidos previamente, reiniciou-se o desmame segundo o protocolo (2ppm por hora), sendo mantidos os valores pressóricos desejáveis.

A internação na Unidade de Terapia Intensiva em média foi entre 12 e 27 dias ( $20 \pm 6$ ), houve um óbito no $17^{\circ}$ dia pósoperatório por sepse e falência de múltiplos órgãos. Os demais pacientes encontram-se atualmente em classe funcional I (NYHA).

O Gráfico 1 demonstra os valores administrados de NOi e a pressão arterial pulmonar em um caso, exemplificando a ação vasodilatadora pulmonar do NO, sem efeito rebote após a redução gradual da concentração do gás inalado.

\section{COMENTÁRIOS}

A crise hipertensiva pulmonar e a disfunção ventricular direita secundária a esta, durante o pós-operatório imediato do transplante cardíaco, continuam sendo preocupações constantes nos centros transplantadores em razão de corresponderem a uma porcentagem elevada dentro das complicações encontradas (até 50\%), e que podem provocar uma alta mortalidade precoce (19\%) ou ainda uma diminuição da vida útil do enxerto ${ }^{(1,2)}$.

O NOi tem se mostrado eficaz no tratamento de situações associadas a quadros de hipertensão pulmonar, tais como no pós-operatório de operações cardíacas com circulação extracorpórea, no tratamento da disfunção do ventrículo direito pós-transplante cardíaco, no peri-operatório do transplante pulmonar, na síndrome de angústia respiratória do adulto, na hipertensão pulmonar persistente do recémnascido, entre outras ${ }^{(4,5,8-10)}$.

O NO, como um vasodilatador pulmonar seletivo, se difunde rapidamente através da membrana alvéolo-capilar, provocando o relaxamento da musculatura lisa do vaso pulmonar. À medida que se difunde dentro da luz do vaso sanguiíneo é fixado pela hemoglobina e inativado, sendo sua metabolização detectada através da metahemoglobinemia, que não deve ultrapassar 3\%. Em razão de sua rápida inativação, as propriedades do NOi estão localizadas na circulação pulmonar, sem produzir o vasorelaxamento sistêmico indesejado ${ }^{(4-6)}$. A ação vasodilatadora do $\mathrm{NO}$ é prontamente interrompida quando este é retirado do circuito ventilatório, desta forma, os efeitos farmacológicos do NO são eliminados em aproximadamente 2 minutos após a interrupção da inalação (Figura 1).

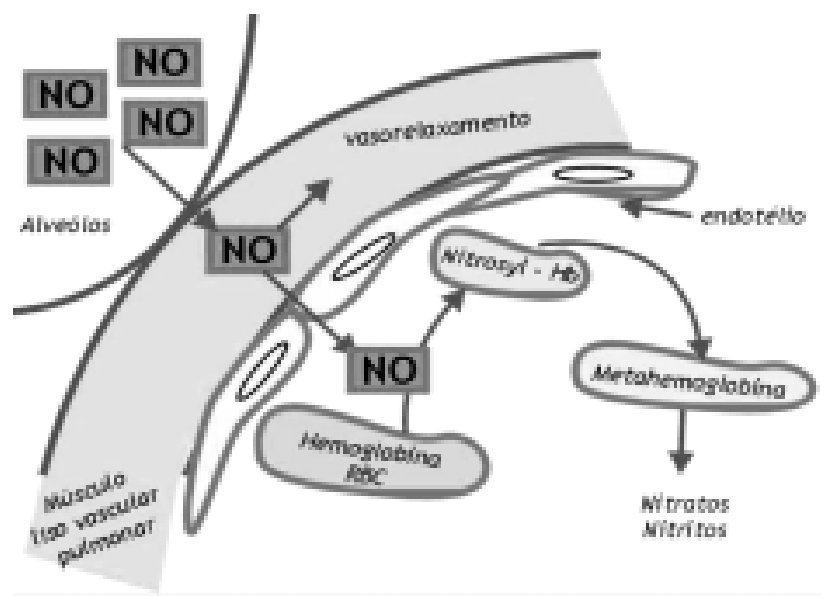

Fig. 1 - Desenho esquemático da ação do NO no alvéolo pulmonar (Baseado em Fullerton et al, 1996).

Outra indicação do NOi foi demonstrada por MEJIA et al. ${ }^{(7)}$ na avaliação hemodinâmica pulmonar em pacientes candidatos à transplante cardíaco e hipertensão pulmonar severa refratária aos vasodilatadores endovenosos convencionais, permitindo que estes fossem incluídos na lista de candidatos.

Estudos têm demonstrado que o NOi quando utilizado em baixas doses (até $80 \mathrm{ppm}$ ) não prejudica a função ventricular esquerda, e este fato pode ser explicado, em parte, pela rápida inativação do NO livre em trânsito ao coração dos pacientes avaliados ${ }^{(7)}$.

AULER et al. ${ }^{(11)}$ demonstraram que, após o transplante cardíaco, o uso do NOi induz vasodilatação pulmonar seletiva, provocando uma redução em 35\% da resistência vascular pulmonar em pacientes com o pós-operatório sem intercorrências. 
Nesta série, foi administrado NOi nos pacientes que não responderam de forma satisfatória ao uso de drogas vasoativas durante o pós-operatório de transplante cardíaco ortotópico frente à CHP. O uso do NO inalatório através do circuito da ventilação mecânica permitiu o manejo desta situação com segurança, quando realizada uma adequada monitorização dos níveis de metahemoglobinemia e NO2 exalado durante seu uso, que, mesmo em situações onde se exigiu um tempo prolongado (96 horas), não foi observado nenhum caso de efeito colateral deletério ou intoxicação. Embora tenha havido um caso de efeito rebote após a suspensão acidental na administração do NOi, o desmame progressivo a $2 \mathrm{ppm}$ por hora demonstrou a manutenção dos efeitos alcançados com o uso do NOi nas doses iniciais.

Ao comparar o grupo dos 5 pacientes que necessitaram do uso de NOi e os demais transplantados, foi identificado um maior tempo de circulação extracorpórea, superior a 120 minutos $(\mathrm{p}<0,001)$. Um tempo prolongado de CEC pode explicar uma maior lesão endotelial pulmonar, provocando redução da síntese de NO e conseqüente aumento da vasoconstricção pulmonar, alterando, desta forma, o equilíbrio do tônus vascular, mesmo em pacientes sem hiperresistência vascular pulmonar prévia. Este efeito foi descrito por BEGHETTI et al. ${ }^{(12)}$, que demonstram níveis exalados de óxido nítrico diminuídos após a circulação extracorpórea relacionados à injúria endotelial pulmonar.

É válido salientar que estes pacientes portadores de doença cardíaca crônica são deficientes na produção de NO endotelial pulmonar, como demonstrado por HART et al. ${ }^{(13)}$ que identificaram, nos pacientes com hipertensão pulmonar, redução da expressão ou ausência da enzima óxido nítrico sintetase, responsável pela síntese endotelial de NO. Isto justificaria o aparecimento de crise hipertensiva pulmonar após CEC prolongada em pacientes com condições pré-operatórias crônicas desfavoráveis, como as encontradas nos candidatos a transplante cardíaco ao se comparar com coronariopatas multiarteriais submetidos a longos períodos de CEC que não apresentam CHP.

NOVITZKY et al. ${ }^{(14)}$, em 1984, e COOPER et al. ${ }^{(15)}$, em 1989, já haviam demonstrado a depressão da função miocárdica após a morte encefálica por alteração metabólica, endócrina e depleção das reservas energéticas do miocárdio. Neste trabalho, ao comparar os 5 pacientes que desenvolveram crise hipertensiva pulmonar no pósoperatório imediato e os demais 26 transplantados, foi identificado um maior tempo de morte encefálica do doador no primeiro grupo, superior a 20 horas $(p<0,001)$. Nestes pacientes, isto pode explicar a falência primária do enxerto consequiente à própria depressão miocárdica pela morte encefálica prolongada, somada aos fenômenos de injúria e reperfusão durante a CEC no transplante cardíaco. Isto justifica um maior tempo de suporte circulatório com CEC, antes da tentativa do desmame da assistência circulatória, que é evidenciado neste estudo como fator de risco para o desenvolvimento da CHP em pacientes transplantados, criando, desta forma, um ciclo vicioso. Esta justificativa é sustentada pela ausência de diferença estatística entre o tempo de anóxia em ambos os grupos de pacientes $(\mathrm{p}=0,105)$, quando comparado ao tempo de CEC total.

Como descrito na literatura ${ }^{(16)}$, neste trabalho não foi possível demonstrar correlação significativa entre valores elevados de resistência vascular pulmonares pré-transplante e o desenvolvimento de crise hipertensiva pulmonar no pósoperatório do transplante cardíaco ortotópico. Deste modo, a ausência de hiper-resistência pulmonar pré-transplante não está diretamente relacionada a uma boa evolução da função pressórica pulmonar durante o pós-operatório imediato do transplante cardíaco. Este conceito reforçaria a necessidade de se manter uma monitorização hemodinâmica contínua com cateter em ateria pulmonar e em átrio esquerdo nos pacientes que apresentem os fatores de risco demonstrados neste trabalho. Isto possibilitaria o diagnóstico precoce da CHP e uma indicação precisa do uso do NOi.

\section{CONCLUSÕES}

Neste trabalho pode-se concluir que tempo de circulação extracorpórea superior a 120 minutos e o tempo de morte encefálica do doador superior a 20 horas sugerem constituir fatores de risco para a crise hipertensiva pulmonar e disfunção do ventrículo direito secundária no pósoperatório imediato do transplante cardíaco.

O óxido nítrico inalatório é uma ferramenta útil, simples e segura no manuseio da crise hipertensiva pulmonar refratária ao tratamento convencional, promovendo redução sustentada, mesmo após o desmame, dos níveis pressóricos da artéria pulmonar. Entretanto, deve-se realizar uma retirada lenta e progressiva para se evitar um possível efeito rebote da pressão arterial pulmonar.

Ante a presença de tempo prolongado de CEC e enxerto de um doador com tempo de morte encefálica elevado, devese colocar cateter em átrio esquerdo e artéria pulmonar para realizar uma monitorização contínua e diagnosticar precocemente casos de CHP.

\section{REFERÊNCIAS BIBLIOGRÁFICAS}

1. Bourge R C, Kirklin J K, Naftel D C, White C, Mason D A, Epstein A E - Analysis and predictors of pulmonary vascular resistance after cardiac transplantation. J Thorac Cardiovasc Surg 1991; 101: 432-45.

2. Wahlers T H, Beer C, Fieguth H G et al. - Right heart failure following orthotopic cardiac transplantation. Clin Transplant $1988 ; 2: 252-6$

3. Fullerton D A \& McIntyre R C - Inhaled nitric oxide: therapeutic applications in cardiothoracic surgery. Ann Thorac Surg 1996; 61: 1856-64. 
4. Bender K A, Alexander J A, Enos J M, Skimming J W - Effects of inhaled nitric oxide in patients with hypoxemia and pulmonary hypertension after cardiac surgery. Am J Crit Care 1997; 6: 127-31.

5. Nonami Y - The role of nitric oxide in cardiac surgery. Surg Today 1997; 27: 583-92.

6. Bacha E A \& Head C A - Use of inhaled nitric oxide for lung transplantation and cardiac surgery. Respir Care Clin N Am 1997; 3: 521-36.

7. Mejia J A C, Pinto Jr. V C, Barroso H B et al. - Baixas doses de óxido nítrico na seleção dos pacientes candidatos a transplante cardíaco com hipertensão pulmonar. Rev Bras Cir Cardiovasc 2001; 16: 28-34.

8. McCarthy P M \& Stinson E B - Routine posttransplant procedures and early postoperative problems after cardiac transplantation. In: Smith J A, McCarthy P M, eds. The Stanford Manual of Cardiopulmonary Transplantation. New York: Futura, 1996: 63-78.

9. Riedel B - The pathophysiology and management of perioperative pulmonary hypertension with specific emphasis on the period following cardiac surgery. Int Anesthesiol Clin 1999; 37: 55-79.
10. Carrier M, Blaise G, Bélisle S et al. - Nitric oxide inhalation in the treatment of primary graft failure following heart transplantation. J Heart Lung Transplant 1999; 18: 664-7.

11. Auler Jr. JO, Carmona MJC, Bocchi EA et al. Low doses inhaled nitric oxide in heart transplant recipients. J Heart Lung Transplant 1996; 15: 443-50.

12. Beghetti M, Silkoff P E, Caramori M, Holtby H M, Slutsky A $\mathrm{S}$, Adatia I - Decreased exhaled nitric oxide may be a marker of cardiopulmonary bypass-induced injury. Ann Thorac Surg 1998; 66: 532-4.

13. Hart C M - Nitric oxide in adult lung disease. Chest 1999; 115:1407-17.

14. Novitzky D, Wincomb W N, Cooper D K C, Rose A G, Fraser R C, Barnard C N - Electrocardiographic hemodynamic an endocrine changes occurring during experimental brain death in the Chacma baboon. J Heart Transplant 1984; 4: 63-9.

15. Cooper D K, Novitzky D, Wicomb W N - The pathophysiological effects of brain death on potential donor organs, with particular reference to the heart. Ann R Coll Surg Engl 1989; 71: 261-6.

16. Hunt S A, Baldwin J, Baumgartner W et al. - Cardiovascular management of a potential heart donor: a statement from the Transplantation Committee of the American College of Cardiology. Crit Care Med 1996; 24:1599-601. 